$5-15-2020$

\title{
Recurrence Relations for Marginal and Joint Moment Generating Functions of Topp-Leone Generated Exponential Distribution based on Record Values and its Characterization
}

\author{
Zaki Anwar \\ Aligarh Muslim University \\ Neetu Gupta \\ Aligarh Muslim University,ngb5433@gmail.com \\ Mohd Akram Raza Khan \\ Aligarh Muslim University \\ Qazi Azhad Jamal \\ Aligarh Muslim University, qaziazhadjamal@gmail.com
}

Follow this and additional works at: https://digitalcommons.wayne.edu/jmasm

Part of the Applied Statistics Commons, Social and Behavioral Sciences Commons, and the Statistical Theory Commons

\section{Recommended Citation}

Anwar, Zaki; Gupta, Neetu; Khan, Mohd Akram Raza; and Jamal, Qazi Azhad (2020) "Recurrence Relations for Marginal and Joint Moment Generating Functions of Topp-Leone Generated Exponential Distribution based on Record Values and its Characterization," Journal of Modern Applied Statistical Methods: Vol. 18 : Iss. 1 , Article 25.

DOI: $10.22237 / \mathrm{jmasm} / 1571545660$

Available at: https://digitalcommons.wayne.edu/jmasm/vol18/iss1/25

This Regular Article is brought to you for free and open access by the Open Access Journals at DigitalCommons@WayneState. It has been accepted for inclusion in Journal of Modern Applied Statistical Methods by an authorized editor of DigitalCommons@WayneState. 


\section{Recurrence Relations for Marginal and Joint Moment Generating Functions of Topp-Leone Generated Exponential Distribution based on Record Values and its Characterization}

Zaki Anwar

Aligarh Muslim University India

Mohd. Akram Raza Khan

Aligarh Muslim University India

\author{
Neetu Gupta \\ Aligarh Muslim University \\ India \\ Qazi Azhad Jamal \\ Aligarh Muslim University \\ India
}

The exact expressions and some recurrence relations are derived for marginal and joint moment generating functions of $k^{\text {th }}$ lower record values from Topp-Leone Generated (TLG) Exponential distribution. This distribution is characterized by using the recurrence relation of the marginal moment generating function of $k^{\text {th }}$ lower record values.

Keywords: $\mathrm{k}^{\text {th }}$ lower record values, Topp-Leone generated exponential distribution, marginal moment generating function, joint moment generating functions, recurrence relations and characterization.

\section{Introduction}

The Topp-Leone (TL) distribution was introduced by Topp and Leone (1955). Nadarajah and Kotz (2003) obtained algebraic expressions of the hazard rate and moments. The density and distribution function of TL distribution is given by

$$
\begin{array}{ll}
F(x)=[x(2-x)]^{\alpha}, & 0 \leq x<1,0<\alpha<1, \\
f(x)=2 \alpha x^{\alpha-1}(1-x)(2-x)^{\alpha-1}, & 0 \leq x<1,0<\alpha<1 .
\end{array}
$$

doi: 10.22237/jmasm/1571545660 Accepted May 27, 2017; Published May 15, 2020.

Correspondence: Qazi Azhad Jamal, qaziazhadjamal@gmail.com 


\section{ZAKI ANWAR ET AL.}

Properties of this distribution, such as the distributions of sums, products and ratios (Zhou, 2006); behaviour of kurtosis (Kotz \& Seir, 2007); record values (Zghoul, 2011); moments of order statistics (Genc, 2012); stress strength reliability (Khan \& Arshad, 2016) were examined. The TL distribution is widely applicable and useful distribution, but because of the restriction on its support set $(0<x<1)$ and having only one parameter, it cannot be used for lifetime modeling.

To overcome these drawbacks, a generalization of TL distribution was presented by Razaei et al. (2017) by using a baseline distribution function as $[G(x, \xi)]^{\theta}$ in Topp-Leone distribution. This new family of distributions is called the Topp-Leone generated family of distributions. The support set of this family of distributions is on the real line $\mathfrak{R}$ and also having one extra parameter makes this distribution very flexible, making it useful for lifetime modeling problem.

Consider $G(x / \theta)$ as the baseline distribution function of scale family where $\theta$ is a scale parameter. The distribution function of the Topp-Leone generated family of distributions is

$$
F(x)=[G(x / \theta)(2-G(x / \theta))]^{\alpha}, \quad \alpha>0, \theta>0, x \in \Re .
$$

The exponential distribution is a special case of $G(x / \theta)$ with distribution function (df)

$$
G(x / \theta)=1-e^{-x / \theta}, \quad \theta>0, x>0 .
$$

The Topp-Leone Generated Exponential (TLG -Exponential) distribution is given as

$$
\begin{array}{ll}
F(x)=\left(1-e^{-2 x / \theta}\right)^{\alpha}, & \alpha>0, \theta>0, x>0, \\
f(x)=\frac{2 \alpha}{\theta} e^{-2 x / \theta}\left(1-e^{-2 x / \theta}\right)^{\alpha-1}, & \alpha>0, \theta>0, x>0 .
\end{array}
$$

The relation between $F(x)$ and $f(x)$ is

$$
F(x)=\frac{\theta}{2 \alpha}\left[e^{2 x / \theta}-1\right] f(x)
$$

Record values arise naturally in many real life applications involving data related to economic, sports, weather and life testing problems. The statistical study of record values started with Chandler (1952). Let $\left\{X_{n}, n \geq 1\right\}$ be a sequence of independent and identically distributed (iid) random variables with distribution function $(d f) F(x)$ and probability density function $(p d f) f(x)$. The $j^{\text {th }}$ order statistic of a sample $X_{1}, X_{2}, \ldots$, 
$X_{n}$ is denoted by $X_{j: n}$. For a fixed $k \geq 1$, we define the sequences $\left\{L_{n}^{(k)}, n \geq 1\right\}$ of $k^{\text {th }}$ lower record times of $\left\{X_{n}, n \geq 1\right\}$ as:

$$
\begin{aligned}
& L_{1}^{(k)}=1 \\
& L_{n+1}^{(k)}=\min \left\{j>L_{n}^{(k)}: X_{j: j+k-1}>X_{L_{n}^{(k)}: L_{n}^{(k)}+k-1}\right\} .
\end{aligned}
$$

For $k=1$ and $n=1,2, \ldots$, write $\mathrm{L} L_{n}^{(1)}=L_{n}$. Then $\left\{L_{n}, n \geq 1\right\}$ is the sequence of record times of $\left\{X_{n}, n \geq 1\right\}$. The sequence $\left\{Y_{n}^{(k)}, n \geq 1\right\}$, where $Y_{n}^{(k)}=X_{L^{(k)}}$ is called the sequence of $k^{\text {th }}$ upper record values of $\left\{X_{n}, n \geq 1\right\}$. For convenience, we shall also take $Y_{0}^{(k)}=0$. For $k=1, Y_{n}^{(1)}=X_{L_{n}}, n \geq 1$, which are the record values of $\left\{X_{n}, n \geq 1\right\}$ (Ahsanullah, 1995). Moreover, $Y_{1}^{(k)}=\min \left(X_{1}, X_{2}, \ldots, X_{k}\right)=X_{1: k}$. Then, the of $p d f Y_{n}^{(k)}$ and the joint $p d f$ of $Y_{m}^{(k)}$ and $Y_{n}^{(k)}$ are:

$$
\begin{aligned}
& f_{Y_{n}^{(k)}}(x)=\frac{k^{n}}{(n-1) !}[-\ln F(x)]^{n-1}[F(x)]^{k-1} f(x), \\
& f_{Y_{m}^{(k)}, Y_{n}^{(k)}}(x, y)=\frac{k^{n}}{(m-1) !(n-m-1) !}[-\ln F(x)]^{m-1} \frac{f(x)}{F(x)}[-\ln F(y)+F(x)]^{n-m-1} \\
& \times[F(y)]^{k-1} f(y), x<y, 1 \leq m<n, n \geq 2 .
\end{aligned}
$$

Various recurrence relations of single and product moments of record values are obtained for example: generalized Pareto distribution (Balakrishnan \& Ahsanullah, 1994) and generalized extreme value distribution (Balakrishnan et al. 1993, and others). Recurrence relations for single and product moments of $k^{\text {th }}$ record values are also obtained for different distributions such as the Gumbel distribution (Pawlas \& Syznal, 1998), Inverse Weibull distribution (Pawlas \& Syznal, 2000) and the linear exponential distribution (Saran \& Singh, 2008). Kamps (1998) investigated the importance of recurrence relations of order statistics in characterization.

In the present study, some explicit expressions and recurrence relations are established for the marginal and joint moment generating functions of $k^{\text {th }}$ lower record values from the TLG-Exponential distribution. A characterization of this distribution has also been obtained by using a recurrence relation of marginal moment generating functions.

\section{Relations for Marginal Moments Generating Functions}

First, establish explicit expression for marginal moment generating function of $k^{\text {th }}$ lower record values $M_{X_{L(n) k}}(t)$. Using (2), 


$$
M_{X_{L(n) k}}(t)=\frac{k^{n}}{(n-1) !} \int_{0}^{\infty} e^{t x}[F(x)]^{k-1}[-\ln F(x)]^{n-1} f(x) d x .
$$

By setting $z=[F(x)]^{1 / \alpha}$ in (4),

$$
M_{X_{L(n) k}}(t)=\frac{k^{n}}{(n-1) !} \int_{0}^{1}(1-z)^{-\theta t / 2} z^{k \alpha-1}(-\ln z)^{n-1} d z
$$
On using the Maclaurin's series expansion, $(1-z)^{-\theta t / 2}=\sum_{p=0}^{\infty} \frac{(\theta t / 2)_{p} z^{p}}{p !}$,
where,

$$
(\theta t / 2)_{p}=\left\{\begin{array}{cc}
\theta t / 2(\theta t / 2+1) \ldots \ldots . .(\theta t / 2+p-1), & p=1,2 \ldots \\
1, & p=0
\end{array}\right.
$$

On integrating the expression of (5),

$$
M_{X_{L(n) k}}(t)=k^{n} \alpha^{n} \sum_{p=0}^{\infty} \frac{(\theta t / 2)_{p}}{p !}\left(\frac{1}{k \alpha+p}\right)^{n}, \quad t \neq 0 .
$$

Remark 1 By setting $k=1$ in (6), the explicit expression of marginal moment generating function of lower record values may be deduced from this distribution.

$$
M_{X_{L(n) 1}}(t)=\alpha^{n} \sum_{p=0}^{\infty} \frac{(\theta t / 2)_{p}}{p !}\left(\frac{1}{\alpha+p}\right)^{n}, t \neq 0 .
$$

Theorem 1 For positive integers $i, j=0,1 \ldots$

$$
M_{X_{L(n) k}}^{(j)}(t)\left[1-\frac{\theta t}{2 \alpha k}\right]=M_{X_{L(n-1) k}}^{(j)}(t)-\frac{\theta j}{2 \alpha k} M_{X_{L(n) k k}}^{(j-1)}\left(t+\frac{2}{\theta}\right)+\frac{\theta}{2 \alpha k}\left[j M_{X_{L(n) k}}^{(j-1)}(t)-t M_{X_{L(n) k k}}^{(j)}\left(t+\frac{2}{\theta}\right)\right] .
$$

Proof: From (2),

$$
M_{X_{L(n) k}}(t)=\frac{k^{n}}{(n-1) !} \int_{0}^{\infty} e^{t x}[F(x)]^{k-1}[-\ln F(x)]^{n-1} f(x) d x .
$$

Integrating (8) by taking $[F(x)]^{k-1} f(x)$ as the part to be integrated and rest for differentiation,

$$
M_{X_{L(n) k k}}(t)=M_{X_{L(n-1) k}}(t)-\frac{t}{k} \frac{k^{n}}{(n-1) !} \int_{0}^{\infty} e^{t x}[F(x)]^{k}[-\ln F(x)]^{n-1} d x .
$$

By using the relation (1) and then simplifying the resulting expression, obtain the relations for marginal moment generating function given in (9) 


$$
M_{X_{L(n) k}}(t)\left[1-\frac{\theta t}{2 \alpha k}\right]=M_{X_{L(n-1) k}}(t)-\frac{\theta t}{2 \alpha k} M_{X_{L(n) k}}\left(t+\frac{2}{\theta}\right)
$$

Differentiating equation (9) $j$ times with respect to $t$ and obtain the required result given in (7).

By differentiating both sides of equation (7) with respect to $t$ and then setting $t=0$, obtain the recurrence relations for single moment of $k^{\text {th }}$ lower record values from TLG-Exponential distribution as

$$
E\left(X_{L(n): k}^{j}\right)=E\left(X_{L(n-1): k}^{j}\right)+\frac{\theta j}{2 \alpha}\left\{E\left(X_{L(n): k}^{j-1}\right)-E\left(\varphi\left(X_{L(n): k}\right)\right)\right\},
$$

where

$$
\varphi(x)=x^{j-1} e^{2 x / \theta}
$$

Remark 2 By setting $k=1$ in (9), deduce the recurrence relation for marginal moment generating functions of lower record values from the TLG-Exponential distribution.

\section{Relations for joint moment generating functions}

On using (3), the explicit expression for the joint moment generating function of $k^{\text {th }}$ lower record values is

$$
M_{X_{L(m, n) \cdot k}}\left(t_{1}, t_{2}\right)=\frac{k^{n}}{(m-1) !(n-m-1) !} \int_{0}^{\infty} e^{t_{1} x}[-\ln F(x)]^{m-1} \frac{f(x)}{F(x)} I(x) d x
$$

where,

$$
I(x)=\int_{0}^{x} e^{t_{2} y}[-\ln F(y)+\ln F(x)]^{n-m-1}[F(x)]^{k-1} f(y) d y .
$$

After integrating $I(x)$ and then simplifying,

$$
I(x)=\sum_{a=0}^{\infty} \frac{\left(t_{2} \theta / 2\right)_{a}}{a !}[F(x)]^{a / \alpha+k} \frac{\Gamma n-m}{(a / \alpha+1)^{n-m}}
$$

Substituting the above expression of $I(x)$ in (10) and simplifying the resulting equation, obtain

$$
M_{X_{L(m, n) k}}\left(t_{1}, t_{2}\right)=k^{n} \alpha^{m} \sum_{a=0}^{\infty} \sum_{b=0}^{\infty} \frac{\left(t_{1} \theta / 2\right)_{b}}{b !} \frac{\left(t_{2} \theta / 2\right)_{a}}{a !} \frac{1}{(a / \alpha+1)^{n-m}} \frac{1}{(a+b+k \alpha)^{m}}
$$




\section{ZAKI ANWAR ET AL.}

Remark 3 By setting $k=1$ in eqn (11), deduce the explicit expression for joint moment generating function for lower record values from this distribution as

$$
M_{X_{L(m, n): 1}}\left(t_{1}, t_{2}\right)=\alpha^{m} \sum_{a=0}^{\infty} \sum_{b=0}^{\infty} \frac{\left(t_{1} \theta / 2\right)_{b}}{b !} \frac{\left(t_{2} \theta / 2\right)_{a}}{a !} \frac{1}{(a / \alpha+1)^{n-m}} \frac{1}{(a+b+\alpha)^{m}} .
$$

Theorem 2 For $1<m<n-2, i, j=1,2 \ldots$

$$
\begin{aligned}
M_{X_{L(m, n) k k}}^{(i, j)}\left(t_{1}, t_{2}\right)\left[1-\frac{\theta t_{2}}{2 \alpha k}\right]=M_{X_{L(m, n-1) \cdot k}}^{(i, j)}\left(t_{1}, t_{2}\right)-\frac{\theta j}{2 \alpha k} M_{X_{L(m, n) k}}^{(j-1)}\left(t_{1}, t_{2}+\frac{2}{\theta}\right) \\
+\frac{\theta}{2 \alpha k}\left[j M_{X_{L(m, n) k}}^{(j-1)}\left(t_{1}, t_{2}\right)-t_{2} M_{X_{L(m, n) k}}^{(j)}\left(t_{1}, t_{2}+\frac{2}{\theta}\right)\right]
\end{aligned}
$$

Proof: For $1 \leq m \leq n-2, i, j=1,2 \ldots$

$$
M_{X_{L(m, n): k}}\left(t_{1}, t_{2}\right)=\frac{k^{n}}{(m-1) !(n-m-1) !} \int_{0}^{\infty} e^{t_{1} x}[-\ln F(x)]^{m-1} \frac{f(x)}{F(x)} G(x) d x,
$$

where,

$$
G(x)=\int_{0}^{x} e^{t_{2} y}[-\ln F(y)+\ln F(x)]^{n-m-1}[F(x)]^{k-1} f(y) d y .
$$

Integrating $G(x)$ by parts and substituting the resulting expression in (13), relations for joint moment generating function given in (14) is

$$
M_{X_{L(m, n) k k}}\left(t_{1}, t_{2}\right)\left[1-\frac{t_{2} \theta}{2 \alpha k}\right]=M_{X_{L(m, n-1) k}}\left(t_{1}, t_{2}\right)-\frac{t_{2} \theta}{2 \alpha k} M_{X_{L(m, n) k}}\left(t_{1}, t_{2}+\frac{2}{\theta}\right)
$$

Differentiating equation (14) $j$ times with respect to $t$ and we get the required result given in (12).

By differentiating both sides of equation (12) with respect to $t$ and then setting $t=0$, we obtain the recurrence relations for product moments of $k^{\text {th }}$ lower record values from TLG-Exponential distribution.

$$
E\left(X_{L(m, n): k}^{i, j}\right)=E\left(X_{L(m, n-1): k}^{j}\right)+\frac{\theta j}{2 \alpha}\left\{E\left(X_{L(m, n): k}^{j-1}\right)-E\left(\varphi\left(X_{L(m, n): k}\right)\right)\right\},
$$

where

$$
\varphi(x, y)=x^{i} y^{j-1} e^{2 y / \theta} .
$$




\section{TOPP-LEONE GENERATED EXPONENTIAL DISTRIBUTION}

Remark 4 By setting $k=1$ in eqn (14), deduce the explicit expression for joint moment generating function of lower record values from this distribution

$$
M_{X_{L(m, n): !}}\left(t_{1}, t_{2}\right)\left[1-\frac{t_{2} \theta}{2 \alpha}\right]=M_{X_{L(m, n-1) !}}\left(t_{1}, t_{2}\right)-\frac{t_{2} \theta}{2 \alpha} M_{X_{L(m, n): 1}}\left(t_{1}, t_{2}+\frac{2}{\theta}\right)
$$

\section{Characterization}

Theorem 3 Let $\mathrm{X}$ be a non-negative random variable having an absolutely continuous distribution function $\mathrm{F}(\mathrm{x})$ with $\mathrm{F}(0)=0$ and $0<\mathrm{F}(\mathrm{x})<1$ for all $\mathrm{x}>0$, then

$$
M_{X_{L(n) ! k}}(t)=M_{X_{L(n-1) \cdot k}}(t)-\frac{\theta t}{2 \alpha k} M_{X_{L(n) \cdot k}}\left(t+\frac{2}{\theta}\right)+\frac{\theta t}{2 \alpha k} M_{X_{L(n) \cdot k}}(t)
$$

If and only if,

$$
F(x)=\left(1-e^{-2 x / \theta}\right)^{\alpha}, \quad x>0, \alpha, \theta>0 .
$$

Proof: The necessary part follows immediately from eqn (9). However, if the recurrence relation in eqn (15) is satisfied then using eqn (2)

$$
\begin{gathered}
\frac{k^{n}}{(n-1) !} \int_{0}^{\infty} e^{t x}[F(x)]^{k-1}[-\ln F(x)]^{n-1} f(x) d x=\frac{(n-1) k^{n}}{k(n-1) !} \int_{0}^{\infty} e^{t x}[F(x)]^{k-1}[-\ln F(x)]^{n-2} f(x) d x-(16 \\
\frac{t \theta}{2 \alpha k} \frac{k^{n}}{(n-1) !} \int_{0}^{\infty} e^{x\left(t+\frac{2}{\theta}\right)}[F(x)]^{k-1}[-\ln F(x)]^{n-1} f(x) d x \\
+\frac{t \theta}{2 \alpha k} \frac{k^{n}}{(n-1) !} \int_{0}^{\infty} e^{t x}[F(x)]^{k-1}[-\ln F(x)]^{n-1} f(x) d x
\end{gathered}
$$

Integrating the first integral on R.H.S. of eqn (16),

$$
\begin{gathered}
\frac{k^{n}}{(n-1) !} \int_{0}^{\infty} e^{t x}[F(x)]^{k-1}[-\ln F(x)]^{n-1} f(x) d x=\frac{t k^{n}}{k(n-1) !} \int_{0}^{\infty} e^{t x}[F(x)]^{k}[-\ln F(x)]^{n-1} d x+ \\
\frac{k^{n}}{(n-1) !} \int_{0}^{\infty} e^{t x}[F(x)]^{k-1}[-\ln F(x)]^{n-1} f(x) d x-\frac{t \theta}{2 k} \frac{k^{n}}{(n-1) !} \int_{0}^{\infty} e^{x\left(t+\frac{2}{\theta}\right)}[F(x)]^{k-1}[-\ln F(x)]^{n-1} f(x) d x \\
+\frac{t}{2 k} \frac{k^{n}(n-1) !}{\int_{0}^{\infty} e^{t x}[F(x)]^{k-1}[-\ln F(x)]^{n-1} f(x) d x} \\
\frac{t k^{n}}{k(n-1) !} \int_{0}^{\infty} e^{t x}[F(x)]^{k-1}[-\ln F(x)]^{n-1}\left\{F(x)-\frac{\theta}{2 \alpha} f(x)\left(e^{2 x / \theta}-1\right)\right\} d x=0 .
\end{gathered}
$$

Applying generalization of the Müntz-Szász Theorem (Hwang \& Lin (1984)) 


\section{ZAKI ANWAR ET AL.}

which proves

$$
\frac{F(x)}{f(x)}=\frac{\theta}{2 \alpha}\left(e^{2 x / \theta}-1\right)
$$

$$
F(x)=\left(1-e^{-2 x / \theta}\right)^{\alpha} \quad x>0, \alpha, \theta>0 .
$$

\section{References}

Balakrishnan, N., \& Ahsanullah, M. (1994). Recurrence relations for single and product moments of record values from generalized Pareto distribution. Communications in Statistics-Theory and Methods, 23(10), 2841-2852. doi: 10.1080/03610929408831419

Balakrishnan, N., Chan, P. S., \& Ahsanullah, M. (1993). Recurrence relations for moments of record svalues from generalized extreme value distribution. Communications in Statistics-Theory and Methods, 22(5), 1471-1482. doi: 10.1080/03610929308831097

Chandler, K. N. (1952). The distribution and frequency of record values. Journal of the Royal Statistical Society. Series B (Methodological), 14(2), 220-228. doi: 10.1111/j.25176161.1952.tb00115.x

Genç, A. İ. (2010). Moments of order statistics of Topp-Leone distribution. Statistical Papers, 53(1), 117-131. doi: 10.1007/s00362-010-0320-y

Hwang, J. S., \& Lin, G. D. (1984). Extensions of Müntz-Szász theorem and applications. Analysis, 4(1-2), 143-160. doi: 10.1524/anly.1984.4.12.143

Kamps, U. (1998). Characterizations of distributions by recurrence relations and identities for moments of order statistics. Handbook of Statistics, 16, 291-311. doi: 10.1016/ s0169-7161(98)16012-1

Khan, M. J. S., \& Arshad, M. (2016). UMVU Estimation of Reliability Function and StressStrength Reliability from Proportional Reversed Hazard Family Based on Lower Records. American Journal of Mathematical and Management Sciences, 35(2), 171-181. doi: 10.1080/01966324.2015.1134363

Kotz, S., \& Seier, E. (2007). Kurtosis of the Topp-Leone distributions. InterStat, 2007(1). Retrieved from http://interstat.statjournals.net/YEAR/2007/articles/0707001.pdf

Nadarajah, S., \& Kotz, S. (2003). Moments of some J-shaped distributions. Journal of Applied Statistics, 30(3), 311-317. doi: 10.1080/0266476022000030084

Pawlas, P., \& Szynal, D. (1998). Relations for single and product moments of kth record values from exponential and Gumbel distributions. Journal of Applied Statistical Science, 7(1), 53-62.

Pawlas, P., \& Szynal, D. (2000). Characterizations of the inverse Weibull distribution and generalized extreme value distributions by moments of kth record values. Applicationes Mathematicae, 27(2), 197-202. doi: 10.4064/am-27-2-197-202

Rezaei, S., Sadr, B. B., Alizadeh, M., \& Nadarajah, S. (2017). Topp-Leone generated family of distributions: Properties and applications. Communications in Statistics-Theory and Methods, 46(6), 2893-2909. doi: 10.1080/03610926.2015.1053935 


\section{TOPP-LEONE GENERATED EXPONENTIAL DISTRIBUTION}

Saran, J., \& Singh, S. K. (2008). Recurrence relations for single and product moments of $\mathrm{k}$-th record values from linear exponential distribution and a characterization. Asian Journal of Mathematics \& Statistics, 1(3), 159-164. doi: 10.3923/ajms.2008.159.164

Topp, C. W., \& Leone, F. C. (1955). A family of J-shaped frequency functions. Journal of the American Statistical Association, 50(269), 209-219. doi: 10.1080/01621459.1955.10501259

Zghoul, A. A. (2011). Record values from a family of J-shaped distributions. Statistica, $71(3), 355-365$.

Zhou, M., Yang, D. W., Wang, Y., \& Nadarajah, S. (2006, January). Some j-shaped distributions: sums, products and ratios. In Reliability and Maintainability Symposium, 2006.

RAMS'06. Annual (pp. 175-181). IEEE. doi: 10.1109/rams.2006.1677371 